\title{
ESTRATEGIA DE DIVERSIFICACIÓN PRODUCTIVA EN PERÚ Y SU APLICACIÓN EN EL SECTOR AGRÍCOLA*
}

\author{
Recibido: 21 de septiembre de 2016 - Aprobado: 02 de agosto de 2017 \\ DOI: $10.22395 /$ seec.v20n44a6 \\ Pedro Barrientos Felipa**
}

\section{RESUMEN}

Este artículo expone los antecedentes y el diseño de la estrategia de diversificación productiva a través de la revisión de diversos documentos en donde se analizan los avances logrados. Para los fines del caso se revisaron documentos extranjeros que han investigado acerca de la estrategia y sus consecuencias. El interés por la diversificación tiene sustento en la influencia de la caída de los precios y la demanda internacional de los commodities, los que tienen valor significativo en la balanza comercial de Perú. Del análisis se concluye que falta convencimiento, por parte de los agentes involucrados, para llevar a cabo el plan que se ha diseñado, asumiendo que esto ocurre en los procesos de cambios estructurales.

\section{PALABRAS CLAVE}

Diversificación productiva; macromarketing; marketing país; matriz de exportaciones; productos no tradicionales; Perú.

\section{CLASIFICACION JEL}

M31; M16

\section{CONTENIDO}

Introducción; 1. El concepto de diversificación productiva; 2. La diversificación productiva y su efecto en las exportaciones; 3. La política económica y la diversificación productiva; 4. Los avances de la diversificación productiva en Perú; 5. Conclusiones; Bibliografía.

\footnotetext{
"Este artículo de revisión es resultado del proyecto de investigación titulado "El Plan Nacional de Diversificación productiva y la estrategia marketing país" ejecutado durante el año 2015. La investigación fue auspiciada y financiada por el Instituto de Investigaciones Económicas, Facultad de Ciencias Económicas, Universidad Nacional Mayor de San Marcos, Lima, Perú.

" Economista, Universidad Ricardo Palma, Lima, Perú. Magíster en Administración, Universidad del Pacífico, Lima, Perú. Doctor en Administración de Negocios Globales, Universidad Ricardo Palma, Lima, Perú. Vice Decano de la Unidad de Investigación y Posgrado, profesor principal y miembro del Instituto de Investigaciones Económicas, Facultad de Ciencias Económicas, Universidad Nacional Mayor de San Marcos, Lima, Perú. Dirección postal: Calle Germán Amézaga 375, Edificio de la Facultad de Ciencias Económicas, Universidad Nacional Mayor de San Marcos, Lima, Perú. Teléfono 051-998648480. Correo electrónico: pbarrientosf@unmsm.edu.pe
} 


\title{
PRODUCTIVE DIVERSIFICATION STRATEGY IN PERU AND ITS APPLICATION IN THE AGRICULTURAL SECTOR
}

\section{ABSTRACT}

This article provides background and design of the productive diversification strategy through the revision of several documents in order to analyze the progress achieved. For this purpose, foreign documents which have been investigated about the strategy and its consequences have been reviewed. The interest for diversification is based on the influence of the drop of prices and the international demand of commodities, which have a significant value in Peruvian commercial market. From the analysis, it has been concluded that agents involve lack conviction to execute the plan that has been designed, assuming that this occurs with the processes of structural changes.

\section{KEY WORDS}

Productive diversification; macromarketing; marketing country; export matrix; nontraditional products; Peru.

\section{JEL CLASSIFICATION}

M31; M16

\section{CONTENT}

Introduction; 1 . The concept of productive diversification; 2. Productive diversification and its effect on exports; 3. Economic policy and productive diversification; 4. Progress of productive diversification in Peru; 5. Conclusions; Bibliography.

\section{ESTRATÉGIA DE DIVERSIFICAÇÃO PRODUTIVA NO PERU E SUA APLICAÇÃO NO SETOR AGRÍCOLA}

\section{RESUMO}

Este artigo expõe os antecedentes e o desenho da estratégia de diversificação produtiva por meio da revisão de diversos documentos em que se analisam os avanços atingidos. Para isso, revisaram-se documentos estrangeiros que pesquisam sobre a estratégia e suas consequências. O interesse pela diversificação tem seu respaldo na influência da queda dos preços e da demanda internacional das commodities, as que têm valor significativo na balança comercial do Peru. Da análise, conclui-se que falta conhecimento, por parte dos agentes envolvidos, para realizar o plano desenhado, assumindo que isso ocorre nos processos de mudanças estruturais.

\section{PALAVRAS-CHAVE}

Diversificação produtiva; macromarketing; marketing país; matriz de exportações; produtos não tradicionais; Peru.

\section{CLASSIFICAÇÃO JEL}

\author{
M31; M16
}

\section{CONTEÚDO}

Introdução; 1. Conceito de diversificação produtiva; 2. Diversificação produtiva e seu efeito nas exportações; 3. A política econômica e a diversificação produtiva; 4. Os avanços da diversificação produtiva no Peru; 5. Conclusões; Bibliografia. 


\section{INTRODUCCIÓN}

Para América Latina, China representa un socio estratégico; es un importante comprador de soja, metales (plomo, níquel, estaño, zinc, acero primario, cobre refinado y aluminio) y petróleo. Perrotti $(2015$, p. 48) menciona que la importancia del país asiático radica en que se ubica "como el segundo mayor importador del mundo detrás de los Estados Unidos de América". De otro lado, Jaramillo, Lehmann y Moreno (2009, p. 87) explican que "América Latina es un continente cuya economía es bastante sensible a las condiciones externas, especialmente al crecimiento del resto de las economías, la evolución de las tasas de interés y de los spreads de deuda, además de los precios de los bienes básicos que exporta".

La crisis internacional, en la cual la contracción de la economía china tiene un papel importante, influye en Perú, como consecuencia de la menor exportación de minerales y la disminución de sus precios internacionales. Los resultados exponen la relativa fragilidad de la balanza comercial cuando hay una alta influencia de la producción tradicional. Perú, en el año 2014, pone en discusión el Plan Nacional de Diversificación Productiva, el cual considera importante realizar actividades planificadas y coordinadas acerca de la producción no tradicional. La diversificación productiva es una estrategia macroeconómica que representa una política de Estado que se relaciona con la preocupación acerca de cómo depender menos de los commodities.

El marketing está rebasando el campo limitado a las actividades comerciales de las empresas y ahora se notan impulsos para su aplicación en las decisiones comerciales de los países, pues el marketing permite a los gobiernos tener una mejor relación con sus mercados objetivos internacionales. Es este el campo en donde actúa el macromarketing, que es el estudio del funcionamiento de la sociedad en general y su relación con la economía global. El artículo, haciendo uso de las herramientas de marketing en el campo de la economía, se divide en cuatro temas de exposición: el concepto de diversificación productiva, el efecto en la matriz de exportaciones, su relación con la política económica y los avances de la diversificación productiva en Perú. A través de ellos se exponen el alcance de la estrategia, así como necesidades que la misma plantea. Al final se presentan las principales conclusiones que se obtuvieron con esta investigación.

\section{EL CONCEPTO DE DIVERSIFICACIÓN PRODUCTIVA}

El concepto de diversificación productiva se puede entender como el proceso a través del cual una organización (país, región, empresa) decide añadir nuevos productos (bienes o servicios) a su actual cartera, poniendo énfasis en el mercado 
internacional. Las organizaciones optan por tal acción porque identifican una oportunidad en el mercado en el cual actúan, es decir, existen mercados que no están siendo convenientemente atendidos. De ahí que los países productores, con la seguridad de que cuentan con los suficientes recursos, tangibles e intangibles, para aprovecharlos y también por la potencialidad de su sistema productivo, aplican esta estrategia.

Perú, en términos amplios de producción, se caracteriza por dos líneas de productos para el mercado internacional: los commodities y los denominados productos no tradicionales (PNT), como ocurre con varios países latinoamericanos. Cualquier decisión que se tome acerca de la diversificación tiene que considerar decisiones alrededor de los productos tradicionales y de los no tradicionales. En la realización de negocios internacionales los commodities peruanos representan el 75\% de las exportaciones; es decir, se exportan productos con un nivel mínimo de valor agregado.

Como menciona Minondo (2009) un país tiene que decidir entre elaborar productos de poco a muy sofisticados; por lo tanto, si considera crecer en el futuro tiene que alterar su especialización y transitar a productos con mayor sofisticación. La diversificación es una estrategia riesgosa en opinión de Walker et al. (2005, p. 52) "porque a menudo entraña el aprendizaje de nuevas operaciones y el trato con grupos de clientes con los que no se está familiarizado". Esta estrategia es aplicada por diversas organizaciones para mejorar su comportamiento en los mercados local e internacional, y a través de ella la empresa busca reinventarse al acceder a nuevos mercados.

En el gráfico 1 se observa el alcance de la estrategia, que ocurre cuando se decide crecer con nuevas actividades. La estrategia tiene tres variaciones: integración vertical, diversificación en negocios relacionados y diversificación en negocios no relacionados. Chiavenato y Sapiro (2011) exponen que este es uno de los modelos estratégicos que actúan como herramienta para la toma de decisiones, y coinciden con Walker et al. (2005) respecto a que la estrategia es arriesgada porque no depende de los productos de la organización que han logrado éxito ni de su posición en mercados establecidos; en ocasiones funciona, y en otras, no. La estrategia debe considerar producir lo que los compradores necesitan y no lo que el país está en capacidad de hacer.

En el ámbito macroeconómico, en cada país hay que considerar el riesgo de los cambios de Gobierno ya que cada nuevo gobernante tiene su propia idea de lo que el país debe producir; así aparece el riesgo del oferente, incluso con las propuestas de las fuerzas políticas y económicas del país. De ahí la importancia de establecer una 
política de Estado, un compromiso de las fuerzas políticas y sociales que trascienda el tiempo y las ideologías políticas, de modo que el compromiso permita optar de manera segura por la diversificación como una estrategia.

Gráfico 1: Representación de la matriz de diversificación

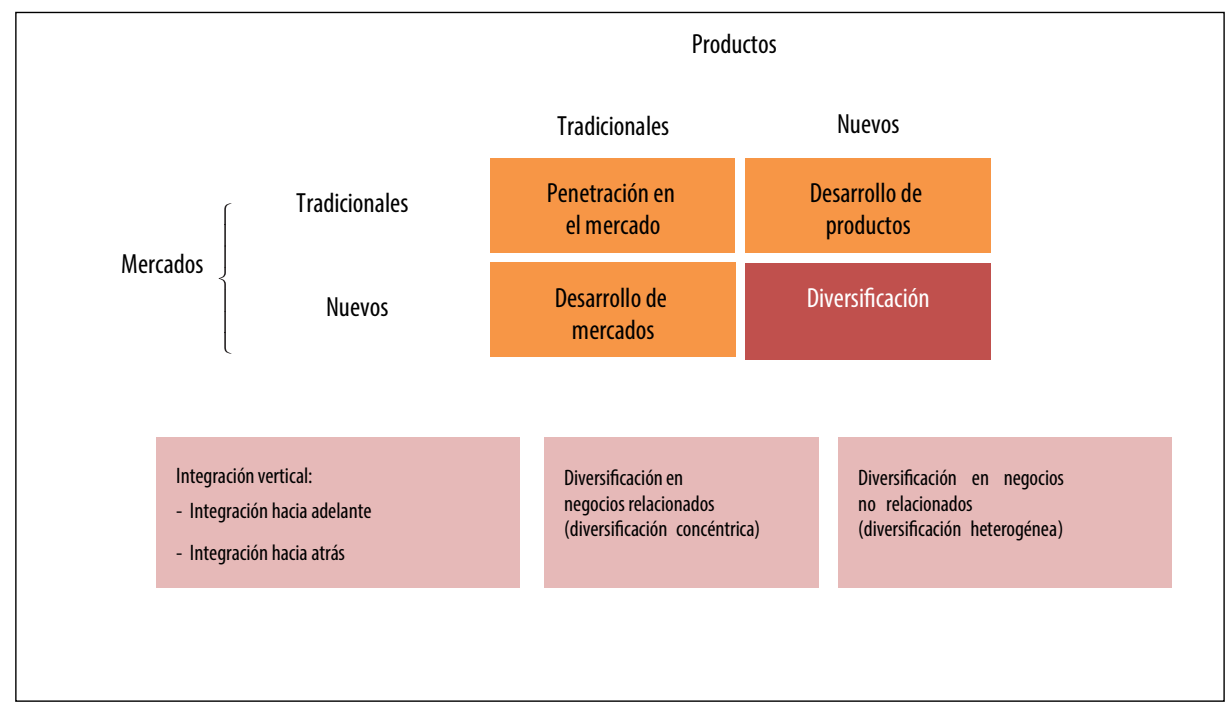

Fuente: Basado en las propuestas de Chiavenato y Sapiro (2011) y Walker et al. (2005)

Se observa, en el gráfico 2, que hay tres opciones de diversificación productiva relacionada (o concéntrica), que incluso pueden combinarse, siendo la etapa superior para un país la de tipo 3. La iniciativa debe fortalecerse con la investigación relacionada con los avances de la ciencia y la tecnología, aplicadas a las actividades, que para el país serán nuevas:

- Diversificación tipo 1. La primera opción es ampliar la cartera de productos, es decir, incrementar la producción actual con nuevos productos en la misma línea, los que antes no se producían y que ahora es razonable producir (se añaden los siguientes productos: P5 y P6). En ambos procesos de producción se mantiene el poco o nulo valor agregado. Por ejemplo, mantener la producción en productos tradicionales, como los minerales.

- Diversificación tipo 2. La siguiente acción es agregar nuevas líneas de productos (P7 y P8) a la actual producción; son bienes que antes no se elaboraban y de sectores diferentes a los productos tradicionales. Se mantiene la característica de productos con poco o nulo valor agregado. El ejemplo es la convivencia de la 
minería y la agricultura (producción no tradicional) que se utiliza como insumo de productos con mayor valor agregado, pero en el lugar de destino.

- Diversificación tipo 3. Se añaden nuevas líneas de productos al comercio internacional que tienen valor agregado, el cual puede otorgarse a las líneas tradicionales existentes (VAP1, VAP2, VAP5 y VAP6); incluso, puede ingresarse a líneas completamente innovadoras (VAP9 y VAP10). Hay una convivencia equilibrada entre productos tradicionales y no tradicionales; productos sin valor agregado y otros que sí lo tienen.

\section{Gráfico 2: Opciones de diversificación productiva}

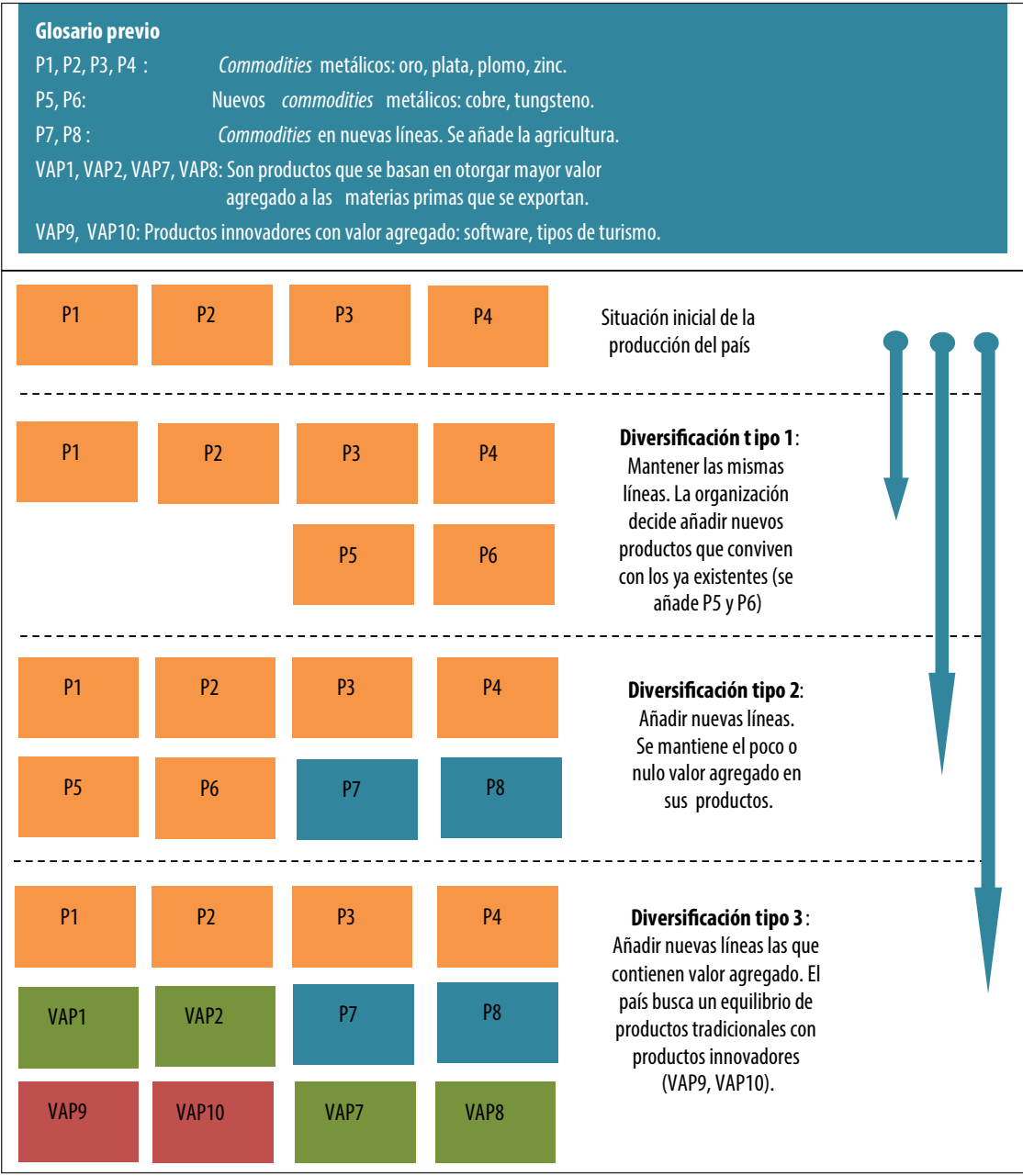

Fuente: elaboración propia 
En una estrategia integral de posicionamiento de país, son importantes los procesos de cadena de abastecimiento y de producción. El país que se compromete con la diversificación para lograr un mejor posicionamiento internacional debe hacer modificaciones en su matriz de producción/exportación, en donde los eslabones de servicios del Estado, el compromiso de las fuerzas políticas y sociales y el compromiso de la inversión privada son estratégicos. Debe considerarse la formación de esta cadena de valor que consolide a las nuevas actividades.

El tema se relaciona con dar una mayor trascendencia en la economía a la clusterización, la formación de cadenas de valor, que encaja muy bien en la estrategia de diversificación, lo cual el Estado debe considerar. Barrientos (2011, p. 55) detalla que "... la aceptación del clúster como una herramienta empresarial corresponde a la adopción de una estrategia de planificación de marketing internacional. Es una actividad de compromiso y está estructurada adecuadamente por la dirección empresarial".

Debe considerarse que la diversificación productiva es un concepto transversal, en la medida que afecta a todos los sectores económicos del país y, por tal motivo, al aceptarse su aplicación en la economía se tiene que convertir en una política de Estado, regulada por una legislación específica.

Minondo (2009) explica que la única forma de favorecer el crecimiento económico es ir trasladando los factores productivos a actividades de mayor sofisticación o darle un mayor valor agregado a la producción actual. Como estrategia de desarrollo de un país, esta presenta riesgos, de modo que puede tener como consecuencia convertirse en un enunciado político de buena intención. Contrario a esto, los inversionistas aceptan el compromiso de ser parte del proceso y para tal caso el Estado debe establecer un marco normativo y un compromiso que permita conocer el papel de los diversos agentes de la economía, de modo que se diseñe un ambiente atractivo que lo haga interesante a la inversión y demuestre que es rentable y sostenible.

Lo que Perú pueda avanzar en la implementación de la estrategia de diversificación productiva (EDP) depende del compromiso que asuman las fuerzas políticas y sociales respecto a qué consideran será el país en las próximas décadas. Las decisiones se toman en un entorno complicado en donde las empresas se enfrentan a una competencia internacional intensa en un mercado que contrae su demanda. Un tema relevante en la diversificación productiva se relaciona con puntos geográficos en los cuales se va a producir. En Perú, hay que identificar los territorios en los cuales se dará mayor énfasis para el desarrollo de la estrategia. Las regiones tienen diferentes ventajas, que pueden ser menos o más favorables a los inversionistas, que otras y como menciona Macías (2004, p. 43) "ellos son uno de los elementos claves 
para entender la posibilidad de una región para atraer inversiones y promover su crecimiento y desarrollo socioeconómico".

Toda actividad económica que realiza un país afecta su posicionamiento: si exporta materias primas se considera primario exportador; ese es su posicionamiento en el comercio internacional; el concepto mismo implica que no se diferencia de otros países. En tal sentido, el marketing-país, o macromarketing, también va por esa ruta, posiciona, reposiciona o desposiciona, siempre con actividades planificadas, no al azar. Todo ocurre en un proceso de largo plazo e implica cambios estructurales de un país en cuánto a qué hacer. A estas acciones se les llama diferenciación.

\section{LA DIVERSIFICACIÓN PRODUCTIVA Y SU EFECTO EN LAS EXPORTACIONES}

La economía peruana, como la mayoría de las economías latinoamericanas, tiene como característica ser proveedora de materias primas para el mercado internacional, lo que se conoce como país primario exportador. Un país exportador de commodities o productos tradicionales se convierte en una economía frágil a los cambios imprevistos de los precios internacionales de estos. En la tabla 1 se observa que del total de exportaciones que realiza Perú, en promedio, el 75\% corresponde a commodities teniendo una participación importante los productos mineros, que en los últimos años han visto caer sus precios en el mercado internacional. Luego, siguen los productos pesqueros y agrícolas, estos últimos con una cada vez menor significancia estadística en las exportaciones.

Tabla 1. Distribución de las exportaciones FOB por grupos de productos (\%)

\begin{tabular}{|l|c|c|c|c|c|c|c|c|c|c|c|c|}
\hline Año & 2003 & 2004 & 2005 & 2006 & 2007 & 2008 & 2009 & 2010 & 2011 & 2012 & 2013 & 2014 \\
\hline Pesqueros & 9,0 & 8,6 & 7,5 & 5,6 & 5,2 & 5,8 & 6,2 & 5,3 & 4,6 & 4,9 & 4,0 & 4,4 \\
\hline Agrícolas & 2,5 & 2,5 & 1,9 & 2,4 & 1,6 & 2,2 & 2,3 & 2,7 & 3,6 & 2,3 & 1,8 & 2,1 \\
\hline Mineros & 51,6 & 55,6 & 56,4 & 61,8 & 62,1 & 58,4 & 60,9 & 61,2 & 59,4 & 57,9 & 55,5 & 52,0 \\
\hline $\begin{array}{l}\text { Petróleo y deri- } \\
\text { vados }\end{array}$ & 6,8 & 5,0 & 8,8 & 7,6 & 8,2 & 8,6 & 7,1 & 8,6 & 9,8 & 10,5 & 12,3 & 11,5 \\
\hline Tradicionales (XT) & $\mathbf{6 9 , 9}$ & $\mathbf{7 1 , 7}$ & $\mathbf{7 4 , 6}$ & $\mathbf{7 7 , 4}$ & $\mathbf{7 7 , 1}$ & $\mathbf{7 5 , 0}$ & $\mathbf{7 6 , 5}$ & $\mathbf{7 7 , 8}$ & $\mathbf{7 7 , 4}$ & $\mathbf{7 5 , 6}$ & $\mathbf{7 3 , 6}$ & $\mathbf{7 0 , 0}$ \\
\hline $\begin{array}{l}\text { No Tradicionales } \\
\text { (XNT) }\end{array}$ & $\mathbf{2 8 , 8}$ & $\mathbf{2 7 , 2}$ & $\mathbf{2 4 , 6}$ & $\mathbf{2 2 , 2}$ & $\mathbf{2 2 , 5}$ & $\mathbf{2 4 , 4}$ & $\mathbf{2 2 , 9}$ & $\mathbf{2 1 , 5}$ & $\mathbf{2 1 , 9}$ & $\mathbf{2 3 , 6}$ & $\mathbf{2 5 , 8}$ & $\mathbf{2 9 , 5}$ \\
\hline Otros & $\mathbf{1 , 3}$ & $\mathbf{1 , 1}$ & $\mathbf{0 , 8}$ & $\mathbf{0 , 4}$ & $\mathbf{0 , 4}$ & $\mathbf{0 , 6}$ & $\mathbf{0 , 6}$ & $\mathbf{0 , 7}$ & $\mathbf{0 , 7}$ & $\mathbf{0 , 8}$ & $\mathbf{0 , 6}$ & $\mathbf{0 , 6}$ \\
\hline TOTAL & 100,0 & 100,0 & 100,0 & $\mathbf{1 0 0 , 0}$ & 100,0 & 100,0 & 100,0 & 100,0 & 100,0 & 100,0 & 100,0 & 100,0 \\
\hline
\end{tabular}

Fuente: Banco Central de Reserva del Perú (2014) 
El valor relativo de las exportaciones no tradicionales (XNT) llega a ser el 25,0\% del total exportado, en promedio, para el período 2001-2014. En el año 2014 representan el 29,5\% del total exportado, la mejor tasa porcentual del período en evaluación, lo que se debe a un menor monto de exportaciones tradicionales (XT) y un pequeño incremento en las XNT (tabla 1). Se menciona el leve crecimiento en las XNT porque estas se mantienen en un nivel similar desde 2012. La principal razón del crecimiento relativo de las XNT es la disminución de las exportaciones de commodities: en 2012 se llega a la cifra de 35.869 millones de US\$, y en 2014 a 27.686 millones de US\$ (tabla 3). Es el riesgo que el país asume cuando su producción y exportaciones se canalizan a los commodities. Decidir por la diversificación productiva optando por nuevos productos, nuevas industrias, es oportuno para disminuir el riesgo de los commodities, aunque no lo evita, porque hay otros factores del entorno que afectan todo proceso económico.

Tabla 2. Exportaciones FOB productos no tradicionales (millones de US\$), 2003-2009

\begin{tabular}{|c|c|c|c|c|c|c|c|}
\hline & 2003 & 2004 & 2005 & 2006 & 2007 & 2008 & 2009 \\
\hline 1. Productos tradicionales & 6.356 & 9.199 & 12.950 & 18.461 & 21.666 & 23.266 & 20.720 \\
\hline Pesqueros & 821 & 1.104 & 1303 & 1.335 & 1.460 & 1.797 & 1.683 \\
\hline Agrícolas & 224 & 325 & 331 & 574 & 460 & 686 & 634 \\
\hline Mineros & 4.690 & 7.124 & 9.790 & 14.735 & 17.439 & 18.101 & 16.482 \\
\hline Petróleo & 621 & 646 & 1.526 & 1.818 & 2.306 & 2.681 & 1.921 \\
\hline 2. Productos no tradicionales & 2.620 & 3.479 & 4.277 & 5.279 & 6.313 & 7.562 & 6.196 \\
\hline Agropecuarios & 624 & 801 & 1.008 & 1.220 & 1.512 & 1.913 & 1.828 \\
\hline Pesqueros & 205 & 277 & 323 & 433 & 500 & 622 & 518 \\
\hline Textiles & 823 & 1.092 & 1.275 & 1.473 & 1.736 & 2.026 & 1.495 \\
\hline Maderas y papeles & 172 & 214 & 261 & 333 & 362 & 428 & 336 \\
\hline Químicos & 316 & 415 & 538 & 602 & 805 & 1.041 & 838 \\
\hline Minerales no metálicos & 74 & 94 & 118 & 135 & 165 & 176 & 148 \\
\hline Siderometalúrgicos & 262 & 391 & 493 & 829 & 906 & 909 & 571 \\
\hline Metal-mecánicos & 99 & 136 & 191 & 164 & 220 & 328 & 369 \\
\hline Otros & 45 & 58 & 70 & 89 & 107 & 121 & 94 \\
\hline 3. Otros & 114 & 131 & 141 & 91 & 114 & 190 & 154 \\
\hline 4. Total exportaciones & 9.091 & 12.809 & 17.368 & 23.830 & 28.094 & 31.018 & 27.071 \\
\hline
\end{tabular}

Fuente: Banco Central de Reserva del Perú (2014) 
Pedro Barrientos Felipa

Tabla 3. Exportaciones FOB productos no tradicionales (millones de US\$), 2009-20014

\begin{tabular}{|l|c|c|c|c|c|c|}
\hline & 2009 & 2010 & 2011 & 2012 & 2013 & 2014 \\
\hline 1. Productos tradicionales & $\mathbf{2 0 . 7 2 0}$ & $\mathbf{2 7 . 8 5 0}$ & $\mathbf{3 5 . 8 9 6}$ & $\mathbf{3 5 . 8 6 9}$ & $\mathbf{3 1 . 5 5 3}$ & $\mathbf{2 7 . 6 8 6}$ \\
\hline Pesqueros & 1.683 & 1.884 & 2.114 & 2.312 & 1.707 & 1.731 \\
\hline Agrícolas & 634 & 975 & 1.689 & 1.095 & 786 & 847 \\
\hline Mineros & 16.482 & 21.903 & 27.526 & 27.467 & 23.789 & 20.545 \\
\hline Petróleo & 1.921 & 3.088 & 4.568 & 4.996 & 5.271 & 4.562 \\
\hline 2. Productos no tradicionales & $\mathbf{6 . 1 9 6}$ & $\mathbf{7 . 6 9 9}$ & $\mathbf{1 0 . 1 7 6}$ & $\mathbf{1 1 . 1 9 7}$ & $\mathbf{1 1 . 0 6 9}$ & $\mathbf{1 1 . 6 7 7}$ \\
\hline Agropecuarios & 1.828 & 2.203 & 2.836 & 3.083 & 3.444 & 4.231 \\
\hline Pesqueros & 518 & 644 & 1.049 & 1.017 & 1.030 & 1.155 \\
\hline Textiles & 1.494 & 1.561 & 1.990 & 2.177 & 1.928 & 1.800 \\
\hline Maderas y papeles & 336 & 359 & 402 & 438 & 427 & 416 \\
\hline Químicos & 838 & 1.228 & 1.655 & 1.636 & 1.510 & 1.515 \\
\hline Minerales no metálicos & 148 & 252 & 492 & 722 & 722 & 464 \\
\hline Siderometalúrgicos & 571 & 949 & 1.130 & 1.301 & 1.320 & 1.152 \\
\hline Metal-mecánicos & 369 & 393 & 476 & 545 & 544 & 581 \\
\hline Otros & 94 & 110 & 147 & 277 & 143 & 161 \\
\hline 3. Otros & $\mathbf{1 5 4}$ & $\mathbf{2 5 4}$ & $\mathbf{3 0 4}$ & $\mathbf{3 4 5}$ & $\mathbf{2 3 8}$ & $\mathbf{1 7 1}$ \\
\hline 4. Total exportaciones & $\mathbf{2 7 . 0 7 1}$ & $\mathbf{3 5 . 8 0 3}$ & $\mathbf{4 6 . 3 7 6}$ & $\mathbf{4 7 . 4 1 1}$ & $\mathbf{4 2 . 8 6 1}$ & $\mathbf{3 9 . 5 3 3}$ \\
\hline
\end{tabular}

Fuente: Banco Central de Reserva del Perú (2014)

En términos de macromarketing, la diversificación productiva es elegir una nueva línea de productos, lo que representa el ingreso a mercados existentes, pero que son nuevos para el país. Hay otras formas de diversificación que implican la posibilidad de la novedad y de que hay mercado para el nuevo producto. Deben considerarse las ideas de Seisdedos (2006), en cuanto a que la diversificación no es producir para vender, sino que el país desarrolla en su estrategia internacional que los clientes (países, personas, empresas) son la parte más importante y que todo esfuerzo debe estar encaminado a diferenciarse en la globalidad del mercado, para lo cual debe identificar diferentes necesidades y plantear soluciones distintas mediante diversas estrategias ad hoc.

Los esfuerzos por diferenciar las exportaciones como país se observan en las tablas 2 y 3, que presentan los resultados de las ventas internacionales (exportaciones) de Perú. Los resultados muestran que se sigue exportando con mayor énfasis 
commodities, pero también evidencia que la agricultura no tradicional está, en el período analizado, aumentando su participación en las exportaciones. En el período 2003-2009 las exportaciones de productos tradicionales se multiplicaron 3,3 veces, llegando a US\$20.720 millones. Los productos no tradicionales se multiplicaron 2,4 veces, llegando a la cifra de US\$ 6.196 millones.

Los precios de los commodities mineros fueron atractivos para las finanzas de los inversionistas, tal como se presenta en la tabla 2, en la que se observa que en el período 2003-2009, las exportaciones mineras pasaron de US\$ 4.690 millones a US\$ 16.482 millones en 2009. En la tabla 3, se observa que el mejor resultado de la producción minera de exportación es en el 2011 cuando llega a un total de exportaciones por US\$27.526 millones que represen el 59,3\% del total de exportaciones. Para Perú, las exportaciones mineras son importantes, pero también son una muestra de su fragilidad, motivo por el cual debe incrementarse el valor relativo de las otras líneas de productos, entre las cuales las de mayor potencial son las del sector agrícola. Es lo que busca el Plan Nacional de Diversificación Productiva (PNDP): mantener la importancia económica de la minería y aumentar la relevancia de los otros sectores exportadores (Ministerio de la Producción, 2014).

El esfuerzo de cambio de la matriz productiva implica que algún organismo del Estado asume el diseño y cumplimiento de la estrategia, el que debe coordinar con todas las instituciones implicadas en el proceso para asegurar que las actividades se interrelacionen. Manzano (2004, p. 90) se pregunta "¿por qué no se diversifican las economías de la región?". Perú también se hace la pregunta, de ahí el establecimiento de una EDP. Entre otras respuestas que Manzano (2004) menciona es que hay ciertos condicionantes macroeconómicos que limitan una decisión relacionada con promover la diversificación, como es el caso de las instituciones, la infraestructura necesaria y la posibilidad de la inserción. También considera el ambiente de negocios, la productividad laboral y la capacidad de innovación de la economía.

En gran parte, lo anterior se relaciona con la educación, la Comisión Económica para América Latina (Cepal, 2015, p. 94) expresa que "la educación es un motor de crecimiento e inclusión social, permite desarrollar las competencias necesarias de su población, y favorece una mayor igualdad de oportunidades". Al considerar el factor humano en el PNDP se logra un punto de apoyo que permite consolidarse de forma sostenible y así tener la suficiente capacidad para que sus tres ejes estratégicos: 1) la diversificación productiva propiamente dicha; 2) la reducción de sobrecostos y de regulaciones, y 3) la expansión de la productividad, permitan lograr el cambio requerido. Este es el sustento del cambio de la matriz exportadora. El Ministerio de Producción (2014, p. 16) explica la relación de los ejes y la orientación hacia el mercado internacional de la manera siguiente: 
La diversificación productiva busca identificar actividades donde existan oportunidades exportadoras latentes para diversificar la canasta exportadora nacional y orientarla a grandes cadenas de valor mundiales. En este eje (en referencia al Eje 1) se buscará -entre otras acciones que se desarrollarán más adelante- expandir las posibilidades tecnológicas mediante el fortalecimiento de las actividades de innovación y emprendimiento innovador.

Queda expuesto, entonces, que es necesario que la matriz de exportaciones se modifique y tal como se expone en el PNDP, hay intención política, es decir, el Gobierno está dispuesto a llevar a cabo tal estrategia. Por tanto, queda asumir el compromiso con y entre los inversionistas, locales y extranjeros, en cómo realizarlo a largo plazo y las acciones que tienen que ejecutarse para el cambio de la producción orientada al comercio internacional. Es así como se integran las actividades de marca país con una estrategia de marketing internacional considerando los grandes agregados económicos, principalmente de las exportaciones, lo que incluye tanto bienes (producción agrícola) como servicios (por ejemplo, turismo).

\section{LA POLIITICA ECONÓMICA Y LA DIVERSIFICACIÓN PRODUCTIVA}

Serino y Mira (2009) manifiestan que las políticas macroeconómicas son las que contribuyen a la diversificación productiva en países que se caracterizan por abundancia de recursos naturales. Y estas políticas son las que deben estar pendientes de la naturaleza dinámica del problema del desarrollo del país. Una economía en la cual las actividades con potencial están lejos de la frontera tecnológica mundial requiere esfuerzos para crear un aumento de productividad suficiente para competir con éxito en la globalización. En este proceso, los países con características económicas similares a Perú también buscan diversificar su producción, considerando la intensidad de la competencia internacional.

La política económica del país como explica Resico (2012, p. 163), coincidiendo con lo que exponen Serino y Mira (2009), sostiene que "el gobierno tiene que orientar su política económica a garantizar un alto nivel de empleo, un nivel de precios estable y un equilibrio del sector externo, así como un crecimiento económico continuo y adecuado", lo que puede lograrse si los diversos gobiernos democráticos se comprometen con la EDP como un proceso de largo plazo, que se construye con la suma de diversas normas legales y compromisos de los actores de la sociedad.

La exportación de materias primas como factor dominante de los negocios internacionales crea barreras al crecimiento de nuevos tipos de producción o de los productos no tradicionales. Es así que el nuevo tipo de producción depende del contexto en que se ubiquen local e internacionalmente, en lo local en cuanto al accionar del Gobierno y los inversionistas (nativos y extranjeros); en lo internacional 
depende de la demanda (empresas en el exterior) y de los competidores que actúan sobre el mismo mercado.

Si se plantea la diversificación productiva como una actividad de macromarketing, tiene que existir una necesidad como país. Porque si algo se crea es que hay una necesidad previa. La necesidad está en los compradores, no en los productores. Hay una diversidad de países (representados por sus empresas) que están en la identificación de la necesidad, todos ellos tienen un punto en común: se relaciona a su dependencia de los commodities. La necesidad está en que los países compradores demandan productos que ellos no producen; la oferta se da por los países que diversifican su producción para aprovechar la oportunidad.

Un problema que tiene la EDP es el definir qué sectores deben ser los prioritarios para realizarla y, por tanto, qué tipo de empresa es la favorecida. Se deben determinar los criterios, porque puede ocurrir que se elija a empresas que tienen mayores recursos financieros y potencialidades que pueden llevar a la marginación de las pequeñas empresas. El proceso requiere de mucho esfuerzo, pues la estrategia tiene que caracterizarse por ser integradora de las empresas peruanas capaces de aceptar el desafío de abordar el mercado internacional, adoptando los procesos y estándares que son requeridos por los compradores.

En el largo plazo la minería seguirá siendo el sector productivo más importante, pero se hace evidente que es necesario un cambio estructural en las exportaciones. Se trata de configurar un progresivo fortalecimiento de las actividades productivas no tradicionales en las que se tengan ventajas comparativas y así aprovechar los cambios en la economía internacional. La apertura económica que hoy se tiene como país debe estar fundamentada sobre cimientos sólidos y esto se puede lograr a través de la diversificación productiva. Barrientos (2011) manifiesta que la complejidad del proceso debe tomar en cuenta la preocupación de la relación del Estado con los inversionistas en cuanto al compromiso para la modificación de la matriz productiva, y de esta manera disminuir la vulnerabilidad en el comercio internacional.

En el 2014, el PIB peruano tuvo una tasa de crecimiento de 2,4\%, producto de los cambios adversos ocurridos principalmente en los países desarrollados, en cuanto a la demanda de minerales; en los cuatro años anteriores (2010-2013) el crecimiento del PIB superó el 5,0\%. La disminución de la demanda por commodities mineros afecta a los países productores y más aún cuando son dependientes de estos. Las exportaciones disminuyeron en $-1,3 \%$ en el 2013 y $-1,0 \%$ en el 2014. El PNDP trata de disminuir la vulnerabilidad de esta dependencia; por tanto, el proceso de las exportaciones debe ser modificado en el largo plazo, con actividades que se realizan en el accionar diario. 
Ser un país primario exportador influye en la imagen internacional del país, y muchos potenciales negocios de mayor valor agregado no pueden ser posibles por esta relación. El marketing considera que las actividades de un país están en relación: 1) con determinados mercados objetivo; y, 2) con el posicionamiento que se busca lograr y, por tanto, se convierte en una herramienta de la dirección de política nacional que puede ser aplicado como en la necesidad a satisfacer en los mercados internacionales.

Cimoli et al. (2005) expresan que la fuente de crecimiento en el largo plazo radica en el progreso técnico, que bien se relaciona con 1) la educación en todos sus niveles, desde el básico hasta el universitario; y, 2) con la transformación de la estructura productiva, que puede incluir las intenciones del PNDP. Los países primario-exportadores deben tener presente lo que exponen Cimoli et al. (2005, p. 32):

La fuente del crecimiento económico en el largo plazo radica en el progreso técnico y en la transformación de la estructura productiva que este promueve. Hacia esta percepción ha convergido la moderna teoría del crecimiento, que rescata la herencia schumpeteriana en su visión del proceso de desarrollo. Analizar el crecimiento en el largo plazo requiere analizar en qué medida el cambio estructural en cada país se ha orientado hacia los sectores que tienen capacidad de promover el progreso técnico, de acompañar las tendencias más dinámicas de la demanda, y generar empleos de alta productividad.

En las tablas anteriores se observa la vulnerabilidad de la economía peruana a los cambios de demanda internacional de commodities; tanto las importaciones como las exportaciones se contrajeron en Perú. Por tanto, la política económica se orienta a hacer las correcciones respectivas y así cumplir con los objetivos que se plantean lo que intenta ser abordado por el PNDP. Lo propuesto en el PNDP lo toma en consideración el Ministerio de Comercio Exterior y Turismo (Mincetur, 2015) en el Plan Estratégico Nacional Exportador (PENX) 2015, en lo que corresponde a la orientación de la producción no tradicional en el comercio internacional, lo que complementa la estrategia PNDP que busca la transformación de la matriz productiva a través de productos no tradicionales exportables. En el PENX 2015, se menciona que Mincetur comparte la estrategia de la diversificación productiva y profundiza la implementación de mecanismos que fortalezcan el desarrollo de exportaciones diversificadas y principalmente no tradicionales.

Ponce y Quispe (2010, p. 248) mencionan que:

En los últimos quince años, la política comercial externa peruana ha seguido dos estrategias claras. Primero, el apoyo dinámico al proceso de negociaciones, que culminó con la firma de acuerdos con los principales socios comerciales y continua en futuras negociaciones con mercados potenciales; y, segundo, el diseño 
Estrategia de diversificación productiva en Perú y su aplicación en el sector agrícola

e implementación de planes estratégicos y operativos a favor de las exportaciones por parte de entidades gubernamentales (Tello, 2008). Estas dos estrategias tienen como objetivo principal dinamizar el comercio exterior con el fin de convertirlo en un motor de desarrollo económico para el país.

Sobre la importancia del comercio internacional, en cuanto a la promoción de las exportaciones, el Consejo Nacional de Competitividad (2014, p. 10) menciona que "... el desarrollo del país y el bienestar de la población requieren que el crecimiento económico en los primeros años sea alto, constante y sostenido. Esto se puede conseguir a través de la consolidación del mercado interno y la conquista de mercados internacionales". Las ideas aquí expuestas se relacionan con el PNDP, motivo por el cual se puede considerar que ambos documentos están articulados en un punto común que la orientación del país hacia el comercio internacional.

\section{LOS AVANCES DE LA DIVERSIFICACIÓN PRODUCTIVA EN PERÚ}

Los objetivos del PNDP son de variada temporalidad y requieren la interrelación de diversos sectores económicos relacionados tanto con el sector público como con el privado. En esta dinámica, el Ministerio de la Producción (2014, p. 44) expone acerca de la institucionalidad del proceso:

El sostenimiento en el tiempo de las intervenciones intersectoriales y territoriales del PNDP exige contar con la institucionalidad adecuada. Ello implica la existencia de tres instancias para la coordinación, el diseño y la implementación de políticas: (1) la Comisión Multisectorial Permanente para la Diversificación Productiva (CMDP), con sus correspondientes grupos técnicos vinculados a cada eje, (2) los propios sectores del Gobierno nacional y los Gobiernos regionales, según sus competencias, y (3) los entes o brazos ejecutores.

El proceso es complejo, pues implica un cambio de cómo un país puede realizar nuevas actividades; por lo tanto, se podrían crear barreras que frenen el proceso. Para su iniciación y desarrollo -en términos de marketing-la diversificación productiva (DP) puede entenderse como la forma de administrar un producto; sin embargo, debe considerarse que es conveniente una mayor promoción del mismo a través de entidades que ayuden al soporte en el largo plazo, tales como en instituciones empresariales, académicas y de investigación.

Ocampo (2011) expresa que la necesidad de una estrategia de desarrollo productivo se sustenta en la estrecha relación que existe entre dinamismo económico y diversificación de las estructuras productivas. Recalca la importancia que tiene el fomento de actividades productivas innovadoras que generen fuertes encadenamientos productivos. Es importante ser innovadores en la creación de nuevas actividades 
productivas, nuevas formas de comercialización, conquistar nuevos mercados, o nuevas formas de estructurar al sector empresarial. Ocampo también menciona que se continúe con la producción y promoción de actividades de exportación tradicionales, pero que esto no sirva de obstáculo a la diversificación productiva. Concluye Ocampo (2011, p 32) que "el manejo apropiado del tipo de cambio a lo largo del ciclo económico es esencial para garantizar este resultado".

El país tiene que enfocarse en aumentar la productividad, no necesariamente a aumentar el valor agregado de cada producto. Se tiene el ejemplo de la agricultura donde se considera, como la mejor vía, industrializar los alimentos; sin embargo, la agro-exportación obtiene mayores ganancias entregando alimentos frescos y con calidad. En ese sentido, el Gobierno debe enfocarse en impulsar al sector privado a crecer en productividad, no en identificar sectores y promoverlos por ellos mismos. Respecto a la diversificación, Ghezzi (2016, p. 43) menciona que se tiene seis líneas de acción principales avanzadas hasta el momento:

a. Implementación de mesas ejecutivas sectoriales para facilitar el surgimiento de nuevos motores.

b. Herramientas no financieras para aumentar la productividad de las mipymes.

c. Herramientas financieras para aumentar la productividad de las mipymes

d. Desarrollo de herramientas e incentivos para impulsar la innovación productiva.

e. Creación del Instituto Nacional de Calidad (Inacal) y fortalecimiento de la infraestructura de calidad para mejorar la productividad del sector privado.

f. Desarrollo y promoción de parques industriales modernos.

El PNDP y los objetivos que ahí se plantean son ambiciosos y necesarios para el país. Se debe considerar que es un proceso de largo plazo, motivo por el cual se convierte en política de Estado e implica el compromiso de las fuerzas políticas, económicas y sociales, así como de las organizaciones internacionales a las cuales Perú está adscrito. Revisar los objetivos del plan demuestra la tarea que hay que realizar y esperar la continuidad del mismo, pues es una opción que no es aislada de lo que ocurre en el mundo, cuya propuesta está basada en muchas experiencias favorables, particularmente en países que tienen características socioeconómicas similares a las de Perú.

Los objetivos que se proponen en el PNDP están relacionados con una mejor y mayor participación en el comercio internacional como pilar del crecimiento económico. La diversificación que se pretende busca identificar actividades de demanda de 
productos para diversificar la canasta exportadora nacional, y orientarla a grandes cadenas de valor mundiales. Considerando la dimensión del desafío, Alarco (2014) plantea que la estrategia y su respectiva implementación pueden ser realizadas a través de la creación del Ministerio de Diversificación Productiva, lo cual no implica que se amplíe la estructura del Estado, sino que se construye sobre la base de otro ministerio existente, relacionado con el comercio exterior y el turismo. A esta nueva institución se le sumarán las funciones de ciencia, tecnología e innovación. La EDP debe tener el suficiente peso político para que las políticas relacionadas se puedan llevar a la práctica.

La tarea pendiente, porque el avance de la estrategia es limitado, a la cual deben llegar los agentes que participen en el proceso de diversificación es lo que corresponde a la identificación de actividades productivas y productos que permitan la modificación exitosa de la producción, considerando este proceso como una acción de largo plazo. Lo complejo de la tarea es asumir de forma consensuada qué actividades de la economía deben ser estimuladas, de modo que permita conocer en qué deben invertir el Estado y el sector empresarial.

Las actividades que se han expuesto en relación con la diversificación productiva como parte de la política macroeconómica, desde el punto de vista del macromarketing, representan la suma de acciones que generan una marca-país, lo cual se construye en el largo plazo como toda actividad que los dirigentes de un país consideran que debe perdurar a favor del bienestar de los ciudadanos. El planteamiento de la diversificación productiva no es novedad en América Latina; lo complicado es convencer a los políticos y los inversionistas de su importancia. Al respecto, Manzano (2004) analiza los alcances y la importancia de la diversificación productiva como factor clave para lograr una mayor presencia en la economía mundial, sin que esto signifique dejar de producir lo que tradicionalmente se viene haciendo.

Ghezzi (2016, p. 80) señala la importancia de la sociedad que debe formar el inversionista privado con el Estado: "El Estado y el mercado son complementarios. El mercado requiere de un Estado eficaz y efectivo que haga respetar los contratos y los derechos de propiedad". Y, en este contexto es conveniente considerar la reflexión de Hidalgo (2009, p. 18) en relación con el papel del Estado, expresado a través de su gobierno:

Finally, these findings motivate a discussion of the role of the government in a country's economy. Much of the debate over the economic role of the public sector has focused on whether the government should be an active economic agent or stay completely out of businesses that are not related to the provision of public goods. Economies are complex evolutionary systems, however, and like all evolutionary systems, are characterized by important path dependencies. 


\section{CONCLUSIONES}

El concepto de diversificación productiva implica la transformación planificada de la estructura productiva en donde los productos no tradicionales tienen un mayor valor relativo en el total de las exportaciones. La PNDP es una estrategia que promueve el incremento del comercio exterior a través de una mayor exportación de productos no tradicionales de modo que estos logren una mayor importancia relativa en la matriz correspondiente. A través de esta acción se busca usar toda la potencialidad de todos aquellos productos e ideas que permitan generar más riqueza y bienestar del país.

Hay hasta tres diferentes tipos de diversificación por los cuales puede optar el productor, desde aquellos en que mantienen su estado natural hasta llegar a un estadio superior en el cual se le agrega valor o se transforma el producto natural, como es el caso de los insumos agrícolas. Cualquiera de los tres modelos que se fomente en cuanto a productos no tradicionales es favorable a la disminución del riesgo de los precios internacionales de los commodities mineros. Perú está en el proceso inicial de pasar del segundo al tercer tipo, lo que toma tiempo para su consolidación.

La matriz de exportaciones de un país con la adopción de la EDP tiende a ser modificada, motivo por el cual se desarrolla un reposicionamiento del país a largo plazo, un desplazamiento de ser un país primario exportador a un país de productos innovadores. El proceso se compromete a asumir nuevos objetivos nacionales por parte de los principales agentes socioeconómicos. Una estrategia de macromarketing, como la EDP, implica optar por nuevas líneas de productos y comprometerse -de acuerdo a sus posibilidades- con otorgar mayor valor a los productos no tradicionales.

La política económica y la diversificación productiva están relacionadas con la dirección del crecimiento económico del país. La política determina el camino por el cual transcurrirá el país, y la diversificación es la herramienta o vehículo con el cual se avanza. La diversificación afecta a todos los sectores de la economía, es decir, es una acción de política de Estado transversal. Los principios que rigen su comportamiento son la guía de los integrantes socioeconómicos, que tienen una orientación hacia la producción de exportación, la misma que tiene ventajas comparativas. Sin embargo, debe buscarse priorizar algunas actividades en donde las fortalezas frente a los competidores internacionales permitan ventajas de largo plazo, entre ellas, la agricultura, la pesca o el turismo, y otras más.

Los avances de la diversificación productiva en el Perú todavía son incipientes, y exponen su complejidad para implementarla, considerando que es un cambio es- 
Estrategia de diversificación productiva en Perú y su aplicación en el sector agrícola

tructural. Las barreras que se pueden crear al proceso vienen de los mismos agentes locales, en cuánto quieren aportar y participar en el proceso. La discusión se puede centrar en políticas económicas, líneas de producción inducidas a la exportación, en productividad de los factores de producción, factores internos que necesitan resolverse o una mezcla de todos ellos. Aunque la aplicación es lenta y se deja a la total iniciativa del inversionista privado, sin considerar un proceso integrador, debe considerarse que la acción política debe tener clara la importancia del cambio de la matriz de exportación; caso contrario se continuará con un crecimiento vegetativo que implica mantener el riesgo de país primario exportador.

\section{BIBLIOGRAFÍA}

Alarco, G. (2014). Diversificación productiva en el Perú: retos y alternativas, p. 219-262. En: Paul Maquet Makedonski Valdeavellano, Armando Mendoza Nava y Ana Romero Cano (eds). Caminos de transición: alternativas al extractivismo y propuestas para otros desarrollos en el Perú, RedGE, ONCE y Fundación Friedrich Ebert , 276p.

Barrientos P. (2011). El clúster en la estrategia de marketing internacional. En: Semestre Económico, Vol. 14, No. 28, p. 49-66.

Chiavenato, I. y Sapiro, A. (2011). Planeación estratégica; fundamentos y aplicaciones. Segunda edición. McGraw-Hill Interamericana Editores S. A., 318p.

Cimoli, M.; Porcile, G.; Primi, A. y Vergara, S. (2005). Cambio estructural, heterogeneidad productiva y tecnología en América Latina, p. 9-39. En: Cimolio, Mario (Ed.) Heterogeneidad estructural, asimetrías tecnológicas y crecimiento en América Latina. BID, NU y CEPAl, 162p.

Ghezzi, P. (2016). Logros y desafíos de la diversificación productiva en el Perú. Lima: Ministerio de la Producción, 85p.

Hidalgo, C. (2009). The dynamics of economic complexity and the product space over a 42 year period. Cambridge: Center for International Development at Harvard University, CID Working Paper, No. 189, 20p.

Jaramillo, P.; Lehmann, S. y Moreno, D. (2009). China, precios de commodities y desempeño de América Latina: algunos hechos estilizados. En: Cuadernos de economía, Vol. 46, p. 67-105.

Macías, A. (2004). Crecimiento económico y competitividad en las regiones. Las ciudades medias de Jalisco: el caso de Zapotlan El Grande. En: Revista Región y Sociedad, Vol. XVI, No. 31, p. $39-82$.

Manzano, O. (2004). Transformación productiva: diversificación productiva y agregación de valor, p. 85-134. En: Castilla, L. M.; Pineda, J.; Scandizzo, S.; Manzano, O.; Ríos, G. y Castilla, L. M. (2004). RED 2004: Reflexiones para retomar el crecimiento. Inserción internacional, transformación productiva e inclusión social (Reporte de Economía y Desarrollo (RED)). Caracas: CAF, 240p.

Minondo, A. (2009). Especialización productiva y crecimiento en los países de renta media. Madrid: Instituto Complutense de Estudios Internacionales, Universidad Complutense de Madrid, 25p. 
Ocampo, J. (2011). Macroeconomía para el desarrollo: políticas anticíclicas y transformación productiva. En: Revista Cepal, No. 104, p. 7-35.

Perrotti, D. (2015). La República Popular China y América Latina: impacto del crecimiento económico chino en las exportaciones latinoamericanas. En: Revista CEPAL, No. 116. p. 47-60.

Ponce, F. y Quispe, M. (2010). Opciones de política económica en el Perú: 2011-2015, p. 235-274. En: Políticas comerciales internacionales y la estructura del comercio exterior peruano. Lima: Fondo Editorial de la Universidad Católica del Perú.

Resico, M. (2012). Introducción a la economía social del mercado. Buenos Aires: Fundación Konrad Adenauer, 384p.

Seisdedos, G. (2006). Creando marca de ciudad: principios básicos. Madrid: Ponencia en el Congreso City Marketing Mayo 2006. 14 p.

Serino, L. y Mira, P. (2009). Diversificación productiva en países con recursos naturales abundantes. Buenos Aires: Ministerio de Economía y Finanzas Públicas, 6p.

Walker, O.; Boyd, H.; Mullins, J. y Larreche, J. (2005). Marketing estratégico; enfoque de toma de decisiones. Cuarta edición. México D.F.: McGraw Hill Interamericana editores S. A., 431p.

\section{OTRAS REFERENCIAS BIBLIOGRÁFICAS}

Banco Central de Reserva del Perú (2014). Memorias Institucionales 2014. Lima: Banco Central de Reserva del Perú, 234 p.

CEPAL -Comisión Económica para América Latina- (2015). Perspectivas económicas de América Latina 2015: educación, competencias e innovación para el desarrollo. Santiago de Chile: Comisión Económica para América Latina, 198p.

Consejo Nacional de la Competitividad (2014). Agenda de Competitividad 2014-2018, rumbo al bicentenario. Lima: Ministerio de Economía y Finanzas, 146p.

Ministerio de la Producción (2014). Plan Nacional de Diversificación Productiva (documento sujeto a consulta pública). Lima: Ministerio de la Producción. 114p.

MINCETUR -Ministerio de Comercio Exterior y Turismo - (2015). Plan Estratégico Nacional Exportador 2025. Lima: Ministerio de Comercio Exterior y Turismo 86p.

SELA -Sistema Económico Latinoamericano y del Caribe- (2012). Influencia de las zonas francas en la diversificación productiva y la inserción de los países de América Latina y el Caribe. Caracas: Sistema Económico Latinoamericano y del Caribe, 50p. 